DOI: 10.20472/EFC.2019.012.016

\author{
LUKÁŠ AUGUSTIN MÁSLO \\ University of Economics, Prague, Czech Republic
}

\title{
POST-KEYNESIAN CONTROVERSY ABOUT UNCERTAINTY: METHODOLOGICAL PERSPECTIVE, PART II
}

\begin{abstract}
:
In this paper, the author follows a discussion of two post-Keynesian economists, Paul Davidson and Rod O'Donnell, about the nature of uncertainty in economics. The author focuses on two points of this discussion: a controversy about possibility/impossibility of such a proof and a criticism of Davidson's allegedly split definition of ergodicity. In a controversy about possibility/impossibility, the author puts O'Donnell to criticism for the latter's reduction of proving to providing empirical evidence and, in effect, omission of extra-empirical cognition. The author accepts O'Donnell's argument of Davidson's split definition and infers his own conclusion: the reason why Davidson keeps ignoring the incompatibility of both definitions of ergodicity is that he does not distinguish cumulative and theoretical probability. The author contends that Davidson's claim about predetermination of long-run outcomes in ergodic processes draws its persuasiveness from the ambiguity of the concept "long run": according to the author, Davidson perceives "long-run" in the meaning of "finitely long" while O'Donnell perceives "long-run" in the meaning of "limit infinity".
\end{abstract}

\section{Keywords:}

ergodicity, uncertainty, probability

JEL Classification: B41, D80 


\section{Introduction ${ }^{1}$}

A tough controversy between Paul Davidson on one side and Rod O'Donnell and other economists on the other side has been underway since 2015. This controversy concerns a conceptualization of the phenomenon of fundamental uncertainty. A central concept of this controversy is the concept of ergodicity.

Ergodicity is a feature of a statistical ensemble meaning that the ensemble (population) displays three characteristics: 1) feature of independence and identity of

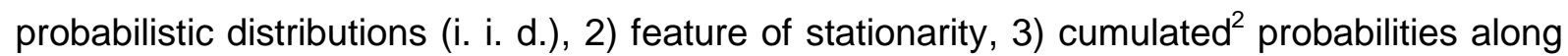
the temporal and spatial axes converge to the same value in the infinity. E. g. the process of coin flipping meets the conditions of ergodicity. Probability that head turns out is the same for each flip of the identical coin. Likewise, this probability is the same for any (fair) coin. Finally, if we flip the same coin again and again, the value of the cumulated probability of this outcome (head) with the number of flips going to infinity will converge to the same value as if we increased the number of coins flipped simultaneously to infinity - in both cases, the value of the cumulated probability of head will converge to 1/2. However, Davidson (2015, pp. 9$10,11,11-12)$ argues that real economic and social processes do not possess the characteristics of ergodicity. ${ }^{3}$ In fact, the probability distribution is changing in time, it does not stay constant as in case of coin flipping. The thing is, in case of a flip of a coin, the probability of head in a single flip is equal to $1 / 2$ independently of the time (or place) in which this flip is being carried out and, at the same time, independently of the cognition and perception of the flipping agent. Probability distribution of states of the world when you are setting up a firm is not independent of cognition and perception of the acting agent, though, because the acting agent affects probabilities of possible outcomes by his action and he affects even the set of these outcomes. Success or fail of a founder of a new firm is not independent of his action as a flip of a coin he is tossing. Development of stock prices may be independent of one particular buyer's action but it is changing with time. Probability that stock prices will go up is not the same in a stage of boom as it is in a stage of recession. Moreover, Davidson (2016, pp. 324-325) stresses that if economic processes were ergodic as flipping a coin - it would mean that all future is predetermined (see below).

According to Davidson, then, real economic and social processes are non-ergodic, and that is why they are dominated by fundamental uncertainty. This uncertainty, Davidson claims, is ontological. Its source is the nature of the world itself and processes therein, supposedly, independently of the cognitive abilities of a man. Now, this is the critical point of Davidson's theory which attracts the main stream of O'Donnell's criticism. The thing is, O'Donnell points to a necessity to prove the claim of the non-ergodic nature of the world which Davidson does not prove. Within the controversy about necessity/unnecessity to

\footnotetext{
${ }_{1}^{1}$ Author: Lukáš Augustin Máslo, University of Economics, Prague, email: lukas.maslo@vse.cz

${ }^{2}$ For repeated flips of a coin, a cumulated probability can be found as a fraction of frequency of the outcome and the number of flips. E. g., if 10 flips produce 3 times head and 7 times tale, the cumulated probability of head is 0,3 . If, afterwards, head turns out 6 times and tale turns out 4 times between the $11^{\text {th }}$ and $20^{\text {th }}$ flip, the cumulated probability of head for the total of 20 flips is $9 / 20$, i. e. 0,45 . For the number of flips going to infinity, the cumulated probability of head should converge to the theoretical probability of this outcome, i. e. 0,5 .

${ }^{3}$ We can imagine an investment into building of a new factory, purchase/sale of stock, decision about starting studies at a university etc.
} 
provide such a proof, a controversy about possibility/impossibility to provide such a proof is going on between O'Donnell and Davidson. O'Donnell insists that the technical definition of ergodicity implies that there is no way to prove ergodicity or non-ergodicity of a process empirically. This is immediately related to O'Donnell's objection to Davidson's allegedly split definition of ergodicity where, apart from the concept of ergodicity in the technical meaning, Davidson uses a substitute definition of ergodicity, allegedly, which meets the conclusions of Davidson's ENE theory better, allegedly, but which does not follow from the technical definition of ergodicity.

This paper does not put forward any hypothesis. The author's main objective is to present the key moments of a discussion between Davidson and O'Donnell. The author's secondary objective is to assess the quality of arguments and to infer his own conclusions. The first section presents a discussion of Davidson and O'Donnell about possibility (Davidson) or impossibility (O'Donnell) to prove ergodicity/non-ergodicity empirically. The second section presents O'Donnell's objection to the alleged splitting of the concept of ergodicity in Davidson's argumentation, along with Davidson's counter-argument based on non-stationarity. The third section presents some consequences of the conclusions of this discussion for the real economy. The summary sums up main points of the discussion and puts forward the author's own assessment.

\section{Possibility/Impossibility Controversy}

As stated above, the discussion about the burden of proof in case of ergodicity/nonergodicity of a process is a by-product of a discussion about possibility/impossibility to provide a proof of ergodicity/non-ergodicity of a process. O'Donnell claims that, with respect to the definition of ergodicity as a limit equality of cumulative probability distributions on the temporal and spatial axes, it is impossible to provide such a proof and, in effect, it is impossible to declare a process ergodic or non-ergodic with certainty:

Agents can only know the true ontological state at infinity, for it is only at infinity that identity or nonidentity of the two sets of statistics [i. e. temporal and spatial cumulative probabilities, L. A. M.] occurs and certain knowledge of ergodicity or nonergodicity is acquired. But infinity never arrives_-knowledge determined at infinity is forever too late, for all agent decisions are necessarily preinfinity. ${ }^{4}$ (O’Donnell, 2014-2015, p. 194)

\footnotetext{
${ }^{4}$ It follows from the very essence of the concept of infinity that this infinity can never be reached. If it could be reached, it would not be infinity. To assert that we know that identity or nonidentity of the two sets of statistics arrives at infinity is therefore pretty much the same as to assert that this identity or nonidentity arrives never. Now this is exactly what O'Donnell's HAC approach to uncertainty stresses: whether an economic agent is acting within an ergodic or non-ergodic process is not so relevant as Davidson's ENE approach suggests - an economic agent is exposed to uncertainty in both cases. By which I do not say that the concept of infinity does not have a content. On the contrary. Philosophy knows the concept of infinity. Infinity is an entity which is not limited by time, space or another entity. It is an ontologically independent entity or the first cause or God. The language of mathematics which deals with accidents cannot express the infinity in a different way than by means of the concept of the limit. In the language of mathematics, then, the concept of infinity evokes something unreal. However, this is given by the limits of this discipline of mathematics.
} 
Let us notice that O'Donnell considers empirics, i. e. experience the only thinkable proof of ergodicity/non-ergodicity. If this were the case, though, we could not say with certainty that a flip of a coin is a case of an ergodic process because this cannot be discovered from experience - with respect to the limit character of ergodicity. Whoever claims, then, that a flip of a coin is a case of an ergodic process - i. e. that a cumulative probability of the outcome $\mathrm{H}^{5}$ at infinity converges to $1 / 2$ with the increase of the number of simultaneously flipped coins and that the cumulative probability of the outcome $\mathrm{H}$ at infinity also converges to $1 / 2$ with the increase of the number of flips of one particular coin - he knows this not from experience but, as Deprez (p. 1240) asserts, from the study of the game itself (see below).

If O'Donnell does not see a substantial - I would say qualitative - difference between uncertainty of ergodic and uncertainty of non-ergodic processes, why does he admonish Davidson for not proving the alleged non-ergodicity of economic processes. What is the point of such an admonition? Is O'Donnell trying to multiply the list of Davidson's fallacies? I do not think so. I can see the following point: the impossibility to prove empirically whether the chance respects or does not respect the theoretical probability distribution of a process at infinity should give the opponent insight that the chance does not need to respect the theoretical probability distribution at all, practically - because the infinity is never reached, by definition. However, since it is exactly the respect of the chance for the theoretical probability distribution what distinguishes ergodic processes from non-ergodic processes and since this moment escapes from us to infinity, this is supposed to be the moment when the opponent gains insight into the fact that the ergodicity/non-ergodicity dichotomy cannot be the criterion for assessment of a character of the uncertainty. A defect of O'Donnell's argument consists in a reduction of the proof of ergodicity to an empirical proof. An empirical proof is not the only way of proving, though (if a term "empirical proof" can be used at all). ${ }^{6}$ The very concept of infinity is not empirical. Nevertheless, this defect is not relevant for the efficacy of O'Donnell's argument, in the end, because this argument's objective was not to disprove the possibility of a proof of ergodicity of a process but to point out the irrelevance of the ergodicity/non-ergodicity dichotomy as a criterion of the fundamental uncertainty. What is to be understood by Deprez's (p. 1240) "study of the game itself"? I contend that it is supposed to be understood as identification of essential characteristics of a process, i. e. characteristics which this process needs to have, as long as this process is supposed to be itself and not a different process. This type of a study of a process (or proving of his ergodicity/non-ergodicity) is not empirical, it does not infer its conclusions from experience.

\footnotetext{
${ }^{5} \mathrm{H}$ for head.

${ }^{6}$ An alternative is a logical proof by contradiction. Let us say that a cumulative probability of the outcome $\mathrm{H}$ with an increasing number of coins to infinity would converge to a different value than the cumulative probability of the outcome $\mathrm{H}$ with the increasing number of flips of a particular coin to infinity. The theoretical probability $\mathrm{P}_{\text {time }}(H)$ representing a proportion of allocation of determining factors in their relation to the outcome in the temporal dimension would then be different from the theoretical probability $\mathrm{P}_{\text {space }}(\mathrm{H})$ representing a proportion of determining factors in their relation to the outcome in the spatial dimension. What would then hold true about the "first" coin in the temporal and also the spatial series? Obviously, it would hold true that the probability $P_{\text {time }}(H)$ in the process of the flip of this coin conceived as a coin from the temporal dimension is different from the probability $\mathrm{P}_{\text {space }}(\mathrm{H})$ in the process of the flip of this coin conceived as a coin from the spatial dimension. Since it is the identical coin, though, the probability of the outcome $H$ would have to be and not to be $P_{\text {time }}(H)$ at the same time; also, the probability would have to be and not to be $\mathrm{P}_{\text {space }}(\mathrm{H})$ at the same time.
} 


\section{Split Term of Ergodicity/Non-Ergodicity}

The controversy about possibility/impossibility of the proof of ergodicity/non-ergodicity is immediately related to the last point of O'Donnell's criticism of Davidson's ENE approach to fundamental uncertainty I would like to mention here and this is Davidson's allegedly split term ergodicity/non-ergodicity. Both problems are two sides of the same coin. O'Donnell (2014-2015, pp. 191-192, 197) claims that Davidson uses the term ergodicity in two meanings, tacitly: 1) in the technical meaning, that is equality of limits of cumulative probability for time and population going to infinity; 2) in the meaning of statistical invariability which I have interpreted at a different point (Máslo, 2016, p. 159) as a continuation in the trend of the cumulative relative frequencies to limit infinity. In the meaning of the statistical invariability, then, ergodicity means a possibility of extrapolation to infinity. In this meaning, then, ergodicity = invariability, non-ergodicity = variability. O'Donnell (2014-2015, p. 196) shows that this second meaning (Davidson's substitute definition) does not follow from the first (technical definition). However, Davidson does not react to this objection in his response (Davidson, 2015) for which he is being admonished by O'Donnell (2016a, p. 18) immediately. Subsequently, O'Donnell (2016b, pp. 146-149) expands his objection. Nevertheless, the only alternative to empirical proving seems to be for O'Donnell that the cognizing agent himself determines the reality and that he has the ontological certainty on these grounds, then. It seems so that O'Donnell suffers from the empiricist fallacy where the source of all cognition is (sensitive) experience ${ }^{7}$ :

Only when the data set generated by the path taken is constrained by artificial and illegitimately imposed patterns can theorists guarantee that ergodicity will only be associated with reducible uncertainty, and nonergodicity with irreducible uncertainty. (O'Donnell, 2016b, p. 149)

Davidson reacts to O'Donnell's objection to the split definition indirectly in his second response on the occasion of reaction to another objection of O'Donnell's, namely that he, Davidson, does not prove ergodicity/non-ergodicity of a process but that he only presupposes it. At last, Davidson breaks through the barrier of the "burden of proof" and, in reference to another paper of his own (Davidson, 1996), he argues that non-ergodicity of a process can be proven by proving the non-stationarity of this process ${ }^{8}$ :

I wrote in my article "Realty [sic] and Economic Theory," "given the empirical evidence that many macroeconomic realizations relations have unit roots and are therefore nonstationary (and since nonstationarity is a sufficient condition for nonergodicity)" (Davidson, 1996, p. 14

\footnotetext{
${ }^{7}$ This philosophical position is self-contradictory because the very thesis which expresses it does not originate in (sensitive) experience. The reason is not that a time series or a particular number are not perceived by our senses. Both the time series and numbers are abstracted quantities which represent a particular sensitive experience, though. The point is that the very thesis represents a reflection of a human cognition. Now, a reflection is not an act of senses but an act of reason. An attempt to eliminate the reason as a source of cognition requires an application of the reason, first, which implies a contradiction. More on this see Fuchs (1995, pp. 101111).

${ }^{8}$ Unlike non-stationarity, stationarity is often simply assumed. See e. g. Vukson (2017, p. 53).
} 
[in the version of the paper published in the JPKE, it is page 494, L. A. M.]), it would appear that there is significant empirical unit roots evidence to indicate that econometric macroeconomic relationships are not generated by an ergodic system! And this unit root evidence is acknowledged before infinity is reached-despite what O'Donnell claims about not knowing if the system is ergodic or not until infinity! (Davidson, 2016, p. 321)

At this point, it is not illegitimate to ask a question whether the non-stationarity which Davidson talks about is non-stationarity of cumulative probability distributions or nonstationarity of theoretical probability distribution. In the former case, such non-stationarity does not prove anything about ergodicity because what is relevant from this viewpoint is only the limit of this non-stationarity for time going to infinity. In the latter case, Davidson would be right. The question is, however, whether non-stationarity of a theoretical probability distribution can be discovered on a finite time series. I doubt about that. I assert, that the reason why Davidson considers his substitute definition of ergodicity compatible with the technical definition is that he does not distinguish cumulative probability distribution from theoretical probability distribution.

\section{Applications}

No matter how philosophical the above discussed controversy between O'Donnell and Davidson about the nature of uncertainty and proof of ergodicity may seem to be, both protagonists are drawing concrete consequences for the real economy from the conclusions of their discussion. Davidson (2016, p. 325) argues that if economic processes were ergodic, the future would be predetermined and so, for example, the government would be unable to affect the real output in a different run but the short run because the real output is predetermined in the long run and, as a result, does not depend on action of the government or economic agents.

Suppose humans have epistemologically based uncertainty even though the future outcomes of the economic system are the result of an ergodic process or even, as in Rosser's argument, a chaos deterministic system. If so, then, in either of these ergodic or deterministic systems, the future is already predetermined. [...] In these cases, nothing the government can do can change the long-run outcome of the economy. (Davidson, 2016, pp. 324-325)

Such an idea can be illustrated as follows. If the real output of the economy is being determined in the long run in a similar way as the cumulative probability of the outcome $\mathrm{H}$ in the process of flip of a coin is being determined in the long run, which is $1 / 2$, then the effort of the government (or economic agents) to reach a different level of the real output in the long run needs to fail in a similar way as an effort of a gambler who wants to get rich by permanently staking his money on the outcome $\mathrm{H}$ in the process of flip of a coin. In a similar way as this gambler breaks even in the long run - even if he can end up in black numbers transitorily in some periods cumulatively (on average) and even though he can end up in red 
numbers transitorily in other periods cumulatively (on average) - the government will not achieve its aim to increase the real output above the predetermined level in the long run, either.

Davidson's conclusion about the predetermination of long-run outcomes in ergodic processes is drawing its persuasiveness from the ambiguity of the term "long run". According to the technical definition of ergodicity, long run means limit infinity in the above given example. The future is only predetermined at infinity, then. Until the infinity is reached, a change is present and it is the time what is a measure of change. As long as time is going on, the infinity has not been reached. The presence of infinity excludes the presence of a change, then, and, in effect, the passing of time. So, is the theoretical probability $1 / 2$ of the outcome $\mathrm{H}$ in the process of a flip of a coin predetermined? There is a saying that the casino always wins. It is not completely true. If a gambler has enough money and if he keeps gambling a sufficiently long time, then, he ends up in black numbers cumulatively (on average) an infinite number of times between the start and the infinity, however small the theoretical probability of his win is. The trouble is that the moments in which the gambler wins cumulatively might be so distant from each other that they lie beyond the budget constraint of a large number of gamblers. If we apply this conclusion to the economic policy, then a government can be satisfied if it makes the average value of the real output for a period stretching from the beginning to the present moment higher than the value of the real output before the beginning, as long as this time period does not exceed its tenure.

If Davidson talks about the predetermined future, I assert that he points to the very fact that the moments in which the gambler (/the government) wins over the casino cumulatively (/achieves an increase in the average value of the real output for the period stretching from the beginning to the present) might be - and usually will be - relatively very distant from each other. Relatively with respect to the time horizon of particular economic agents or the government. So, Davidson perceives the term "long-run" as "finitely long". O'Donnell, on the contrary, stresses the fact that the limit value of the cumulative results of a gambler (/limit value of the average real output for the period stretching from the beginning to the present) at infinity does not have a practical relevance as long as the time is passing because the distances between the moments of the cumulative wins (/moments of the average increase of the real output for the period stretching from the beginning to the present) are unknown, as much as the respective values of these respective cumulative wins (/value of the average real output for the period stretching from the beginning to the present) are unknown. So, O'Donnell perceives the term "long-run" as "limit infinity". Let us remind ourselves, though, that neither Davidson, nor O'Donnell claim that real economic processes are ergodic (as a flip of a coin). What O'Donnell claims is just that even if these processes were ergodic, this would not eliminate the irreducible uncertainty from the economic agents' decision making, since this uncertainty is not ontological but epistemological. Davidson, on the other hand, claims that if the real economic processes were ergodic, the fundamental uncertainty would disappear from the economic agents' decision making and the future would be knowable in advance because it would be predetermined. 


\section{Summary}

O'Donnell argues that, with respect to the definition of ergodicity, it is not possible to provide a proof of ergodicity or non-ergodicity and that it is not possible to declare a process ergodic or non-ergodic with certainty. I was criticizing O'Donnell at this point for reducing a proof of ergodicity/non-ergodicity to the empirical proof and, as a result, elimination of extraempirical sources of cognition which is particularly piquant with respect to the fact that the very concept of infinity, which O'Donnell uses, does not originate in our experience.

The controversy about possibility/impossibility to prove ergodicity/non-ergodicity is immediately related to O'Donnell's objection that Davidson splits the definition of ergodicity when Davidson uses this term - tacitly - in two different, mutually not necessarily compatible meanings: 1) technical meaning, i. e. meaning of equality of limits of cumulative probabilities for time and population going to infinity (technical definition); meaning of statistical invariability (substitute definition). At this point, I showed that the reason why Davidson considers his substitute definition of ergodicity compatible with the technical definition is that he does not distinguish cumulative probability distribution from theoretical probability distribution.

To support his thesis of non-ergodicity of real economic processes, Davidson presents an argument that if economic processes were ergodic, the future would be predetermined and so, for example, the government would be unable to affect the real output in a different run but the short run. I showed, that if Davidson talks about a predetermined future, he points to the fact, that the moments in which the government achieves an increase in the average real output for a period stretching from the beginning to the infinity will usually be very distant from each other. I showed that Davidson perceives the term "long-run" in the meaning of "finitely long". I continued by arguing that O'Donnell, in opposition to Davidson, stresses the fact that the limit value of the average real output for the period stretching from the beginning to the infinity does not have a practical relevance, as long as the time is passing, because the distances between the moments of the average increase in the real output for the period stretching from the beginning to the infinity are unknown. I provided the evidence that O'Donnell perceives the term "long-run" in the meaning of "limit infinity".

I inferred from Davidson - O'Donnell discussion that O'Donnell perceives the difference between probabilistic and non-probabilistic uncertainty as quantitative, rather than qualitative. I showed that for Davidson, on the other hand, the transition from the sphere of probabilistic to the sphere of non-probabilistic uncertainty is a qualitative change, rather than a change in degree. 


\section{References:}

Davidson, P. 1996. Reality and Economic Theory. Journal of Post Keynesian Economics.

Summer 1996, Vol. 18, No. 4, pp. 479-508

Davidson, P. 2015. A rejoinder to O'Donnell's critique of the ergodic/nonergodic explanation of Keynes's concept of uncertainty. Journal of Post Keynesian Economics. Vol. 38, No. 1, pp. 1-18.

Davidson, P. 2016. Rejoinder to Rosser, O'Donnell, and Carrión Álvarez and Ehnts on their criticisms of my ergodic/nonergodic formulation of Keynes's concept of an actuarial certain

future vs. an uncertain future. Journal of Post Keynesian Economics. Vol. 39, No. 3, pp. 308333.

Deprez, J. 2001. Risk, Uncertainty and Nonergodicity in the Determination of Investment-

Backed Expectations: A Post Keynesian Alternative to Posnerian Doctrine in the Analysis of Regulatory Takings. Loyola of Los Angeles Law Review, 4-1-2001, pp. 1221-1254

Fuchs, J. 1995. Filosofie. 2. Kritický problém pravdy. Praha: Krystal OP, 1995, ISBN 80-85929-06-6.

Máslo, L. 2016. Determinismus, path-dependence a nejistota pohledem postkeynesovské ekonomie. Dissertation, University of Economics, Prague.

O'Donnell, R. 2014-2015. A critique of the ergodic/nonergodic approach to uncertainty. Journal of Post Keynesian Economics. Vol. 37, No. 2, pp. 187-209.

O'Donnell, R. 2016a. Second contribution to the ENE critique: Reply to Davidson, part 1. Journal of Post Keynesian Economics. Vol. 39, No. 1, pp. 17-43.

O'Donnell, R. 2016b. Third contribution to the ergodic/nonergodic critique: Reply to Davidson, part 2. Journal of Post Keynesian Economics. Vol. 39, No. 2, pp. 145-171.

Vukson, W. 2017. Intereset Rate Uncertainty and FDI Pre-Globalisation. International Journal of Economic Sciences. Vol. VI, No. 1, pp. 41-78. 